\title{
Painting a picture of protein interaction
}

The binding interface of interacting proteins is revealed by a novel 'protein painting' technique.

Defining contact sites between proteins is important for understanding their function and invaluable in developing therapies that can block undesirable interactions. However, characterizing protein-protein interfaces by existing methods is still a challenging task.

In a recent study, Alessandra Luchini and her colleagues at George Mason University describe a new technique for identifying the functional contact regions between proteins. The methodology is based on 'molecular paints', a set of small-molecule dyes that tightly adhere to the surface of protein complexes but do not penetrate their buried interaction sites. The painted portions of the proteins are protected from digestion by an enzyme, leaving the nonpainted residues that are engaged in protein-protein interaction susceptible to proteolysis. The short peptides that result from digestion are then analyzed by mass spectrometry, revealing the amino acid sequence of the interacting regions.

Luchini's team applied their method to pinpoint functionally important contact sites between proteins in a tripartite complex composed of the cytokine interleukin-1 $\beta$ (IL-1 $\beta$ ), its receptor IL-1R and the accessory protein IL-1RAcP. They identified the socalled hotspots, or residues crucial for interaction between these three proteins, which correlated well with hotspots predicted by different algorithms, thus validating the approach. Furthermore, protein painting outperformed other mass spectrometrybased techniques for mapping the proteinprotein interface residues, such as crosslinking, hydrogen-deuterium exchange and hydroxyl radical footprinting.

Aberrant IL-1 $\beta$ signaling has been implicated in a variety of diseases, including cancer and autoimmune disorders such as rheumatoid arthritis, systemic lupus erythematosus and inflammatory bowel disease. To test the functional significance of protein painting, Luchini's team used the IL-1RAcP interacting sequence to generate two inhibitors of the three-way interaction between the interleukin ligand, its receptor and the accessory protein. These new inhibitors, a peptide and an antibody, efficiently blocked the tripartite-complex formation.

The researchers suggest that protein painting can be used to explore previously uncharacterized interacting regions between proteins or to confirm and extend the knowledge about these interactions gathered by computational or analytical approaches. Vesna Todorovic

\section{RESEARCH PAPERS}

Luchini, A. et al. Protein painting reveals solventexcluded drug targets hidden within native proteinprotein interfaces. Nat. Commun. 5, 4413 (2014). 